\title{
Components of short-horizon individual security returns*
}

\author{
Jennifer Conrad \\ Unicersity of North Carolina, Chapel Hill, NC 27599, USA
}

Gautam Kaul

University of Michigan, Ann Arbor, MI 48109, USA

\author{
M. Nimalendran \\ University of Florida. Gainesrille, FL 32611, USA
}

Received May 1990, final version received January 1991

In this paper, we present a simple model which relates security returns to three components: an expected return, a bid-ask error, and white noise. The relative importance of the various components is empirically assessed, and the model's ability to explain the various time-series properties of individual security and portfolio returns is tested. Time-varying expected returns and bid-ask errors are found to explain substantial proportions (up to 24\%) of the variance of security returns. We also reconcile the typically negative autocorrelation in security returns with the strong positive autocorrelation in portfolio returns.

\section{Introduction}

Recent research into the time-series behavior of short-horizon returns to individual securities and portfolios has revealed some intriguing properties. Conrad and Kaul (1988, 1989), Lo and MacKinlay (1988), and Mech (1990) show that weekly and monthly portfolio returns are strongly positively auto-

\footnotetext{
* We appreciate the comments and suggestions made by Victor Bernard, Harry DeAngelo, Wayne Ferson, Mark Flannery, Michael Gibbons, Campbell Harvey, Bruce Lehmann, M.P. Narayanan, Gregory Niehaus, Nejat Seyhun, Robert Whaley, seminar participants at Duke University and the University of North Carolina, and especially G. William Schwert (the editor) and an anonymous referee. Comments by Eugene Fama, Kenneth French, and Tom George on related work were helpful in clarifying some important issues. We are grateful to Patti Lamparter for preparing the manuscript. Partial financial support for this project was provided by the school of Business Administration, University of Michigan.
} 
correlated, and that the extent of positive autocorrelation is inversely related to firm size. The strong positive autocorrelation in portfolio returns implies that returns are predictable which, in an efficient market, simply reflects time variation in expected returns. However, an interesting aspect of the predictability of stock returns is that it is asymmetric: Lo and MacKinlay (1990) and Mech (1990) show that the stock returns of large firms can be used to predict returns of smaller stocks, but not vice versa. This phenomenon cannot be explained by nonsynchronous trading [see Lo and MacKinlay (1990) and Conrad and Kaul (1991)], and hence should be consistent with any model of security returns.

In contrast to the positive autocorrelation in portfolio returns, Fama (1965, 1976), French and Roll (1986), and Lo and MacKinlay (1988, 1990) find that short-horizon individual security returns tend to be negatively autocorrelated, although the negative autocorrelation is generally much weaker than the positive autocorrelation in portfolio returns. Moreover, the returns of most securities are negatively autocorrelated, but larger firms' stocks tend to exhibit weak positive autocorrelation. For example, French and Roll (1986) show that the first-order autocorrelations of the returns of the largest three quintiles of NYSE and Amex stocks are positive [see also Kaul and Nimalendran (1990)].

In an attempt to reconcile the different time-series properties of portfolio and individual security returns, some researchers [notably Lo and MacKinlay (1988)] suggest that security returns are made up of a positively autocorrelated common component, a negatively autocorrelated idiosyncratic component related to market microstructure effects, and a white-noise component. The tendency of individual security returns to exhibit negative autocovariance suggests that the market microstructure effects dominate the positive autocovariance induced by the common component. On the other hand, idiosyncratic market microstructure effects are diversified away in portfolios, producing strong positive autocorrelation in portfolio returns.

However, no study has investigated the ability of a particular model of security returns to explain the contrasting time-series behavior of security and portfolio returns. Moreover, there is little evidence regarding the relative importance of specific components of returns. In this paper, we present a simple model of returns based on the assumption that an individual security's transaction return $\left(R_{t}\right)$ is made up of three independent components: a positively autocorrelated expected return component $\left(E_{t}\right)$, a negatively autocorrelated bid-ask error component $\left(B_{t}\right)$, and a white-noise component $\left(U_{t}\right)$. We introduce a methodology to extract the 'unobservable' components of security returns, and then empirically assess the relative importance of the various components as well as test the model's ability to explain the various time-series properties of asset returns. 
Using a sample of NASDAQ weekly returns, we show that time-varying expected returns and bid-ask errors together explain substantial proportions (up to $24 \%$ ) of the variance of security returns. Our simple model also captures most of the important time-series characteristics of short-horizon security returns. In particular, we reconcile the negative autocorrelation in security returns with the positive autocorrelation displayed by portfolio returns, and show that our measures of the expected return component of large and small firms reflect the asymmetric lagged cross-correlations uncovered by Lo and MacKinlay (1990) and Mech (1990). Finally, our bid-ask measure displays appropriate time-series properties, and can explain most of the negative autocovariance displayed by security returns.

In section 2 we describe our model for security returns, introduce our measures of the expected return and bid-ask components, and analyze their time-series properties. In section 3 we present estimates of the proportions of variation in security returns due to the expected return, bid-ask error, and white-noise components, and reconcile the different time-series properties of security and portfolio returns. Section 4 concludes with a brief summary.

\section{A simple model for security returns}

Our model is based on the assumption that observed, or transaction, prices are determined from 'true' prices by adjusting for the bid-ask spread. We use the following notation in describing the model:

$P_{t}^{0}=$ logarithm of observed/transaction price of a security at time $t$,

$Q_{t}=$ unobservable indicator for the bid-ask classification of $P_{t}^{0}, Q_{t}=+1$ if transaction at time $t$ is at the ask and $Q_{t}=-1$ if it is at the bid,

$P_{t}=$ 'true' price of a security which reflects all publicly available information at time $t$,

$E_{t}=$ expected return for the period $t-1$ to $t$ based on all public information up to time $t-1$,

$U_{t}=$ adjustment in 'true' prices due to the arrival of public information between period $t-1$ and $t$,

$s$ = bid-ask spread quoted by the market maker (assumed to be constant at least over short intervals).

Our model for transaction prices can then be written as

$$
\begin{aligned}
& P_{t}^{0}=P_{t}+\frac{s}{2} Q_{t}, \\
& P_{t}=E_{t}+P_{t-1}+U_{t} .
\end{aligned}
$$


Let $R_{t}$ be the continuously compounded transaction return from period $t-1$ to $t$. From (1) and (2), it follows that

$$
R_{t}=E_{t}+B_{t}+U_{t}
$$

where

$$
B_{t}=\frac{s}{2}\left[Q_{t}-Q_{t-1}\right]
$$

Hence, an individual security's transaction return, $R_{t}$, is made up of three components: $E_{t}$, which is an expected return component based on all information up to time $t-1, B_{t}$, which is an error component induced by the bid-ask spread, and $U_{t}$, which is a white-noise component due to information released between time $t-1$ and $t$.

\subsection{The expected return component}

The particular process assumed for expected returns is not of critical importance: we only require that the expected return of security $i, E_{i t}$, follows a positively autocorrelated stationary process. Of course, we cannot observe $E_{i t}$. However, the expected return of a portfolio of securities, $E_{p t}$, is readily extractable [see Conrad and Kaul (1988)]. Since $E_{p t}$ exhibits substantial variation through time, it is a natural candidate for extracting $E_{i t}$ from security returns. Accordingly, we estimate a regression of the form

$$
R_{i t}=\alpha_{i p}+\beta_{i p} E_{p t}+\eta_{i t},
$$

where $E_{p t}$ is the expected return of an equal-weighted size-based portfolio to which security $i$ belongs and $\eta_{i t}$ is the disturbance term.

Our methodology to extract the expected return of an individual security is similar in spirit to the procedure used by Gibbons and Ferson (1985), who provide some evidence that even individual securities contain a predictable component. The only assumptions required for least-squares estimators $\hat{\alpha}_{i p}$ and $\hat{\beta}_{i p}$ to be consistent are that: (1) $E_{p t}$ be uncorrelated with the bid-ask error and the noise components, that is, $\operatorname{cov}\left(E_{i t}, B_{i t}\right)=\operatorname{cov}\left(E_{i t}, U_{i t}\right)=0$, and (2) the bid-ask error components of security returns are cross-sectionally uncorrelated, that is, $\operatorname{cov}\left(B_{i t}, B_{j t}\right)=0$. Given these assumptions, the $\beta_{i p}$ of each security in (4) will be primarily determined by the average covariance of security $i$ 's expected return with the expected returns of the remaining securities in portfolio $p$. Since the expected returns of all securities in a particular portfolio (size-based in our case) are likely to be positively correlated, that is, $\operatorname{cov}\left(E_{i t}, E_{j t}\right)>0, E_{p t}$ will extract an estimate of the expected return of a particular security. 
Since $E_{p t}$ is a proxy for $E_{i t}$, the $R^{2}$ obtained from estimates of (4) will provide a lower bound on the degree of variation in security returns due to time-varying expected returns. The $R^{2}$ (denoted by $\rho_{p e}^{2}$ ) will measure the 'true' degree of variation in $E_{i t}$ only under the unlikely condition that the expected returns of all securities are perfectly positively correlated [see Conrad, Kaul, and Nimalendran (1990)]. However, caution must be exercised in interpreting the $R^{2}$ 's because they may suffer from an upward data-snooping bias, since systematic patterns in portfolio returns are assumed to arise solely due to time-varying expected returns.

\subsection{The evidence}

Since the analysis of the components of transaction returns relies on the availability of transaction and bid/ask prices, we primarily use the CRSP daily return files for NASDAQ stocks for the 1983-1987 period. Following French and Roll (1986) we divide the five years into ten six-month subperiods. The weekly return of each security is calculated as the return from Wednesday closing price to the following Wednesday's close. For each week, only the securities that have trade and bid/ask prices available for both Wednesdays are sorted into three portfolios based on market value at the beginning of each six-month subperiod. Weekly holding period returns of securities within each portfolio are equally-weighted to form three series of portfolio returns. Finally, our tests require returns of individual securities which belong to the size-based portfolios for each subperiod. To ensure that we have an unbroken series of returns available for each security in each subperiod, we only retain securities which have trade and bid/ask prices available for all Wednesdays within a particular subperiod. Consequently, the number of firms in each portfolio varies over the ten six-month subperiods.

The descriptive statistics of our NASDAQ sample indicate that our average firms is comparable to the representative firm on the NYSE and Amex. For example, the median market value of firms in our sample is approximatcly $\$ 180$ million, compared to a market value of approximately $\$ 175$ million for the median firm on the NYSE and Amex. The average spread of firms in our sample is $2.91 \%$, compared to an average spread of $2.82 \%$ for NYSE and Amex firms. ${ }^{1}$ Hence, although our sample contains neither very large (like the Dow Jones 30) nor very small firms, our analysis presumably extends to the representative firm in the stock market.

Average estimates of the parameters of (4) for NASDAQ firms in the three portfolios are reported in table 1. Estimates of $E_{p t}$ used in (4) are obtained by constructing parsimonious time-series models for portfolio realized re-

\footnotetext{
'The descriptive statistics of NYSE and Amex firms are based on December 1988 values provided very kindly by Dunald Keim [see also Keim (1989)].
} 


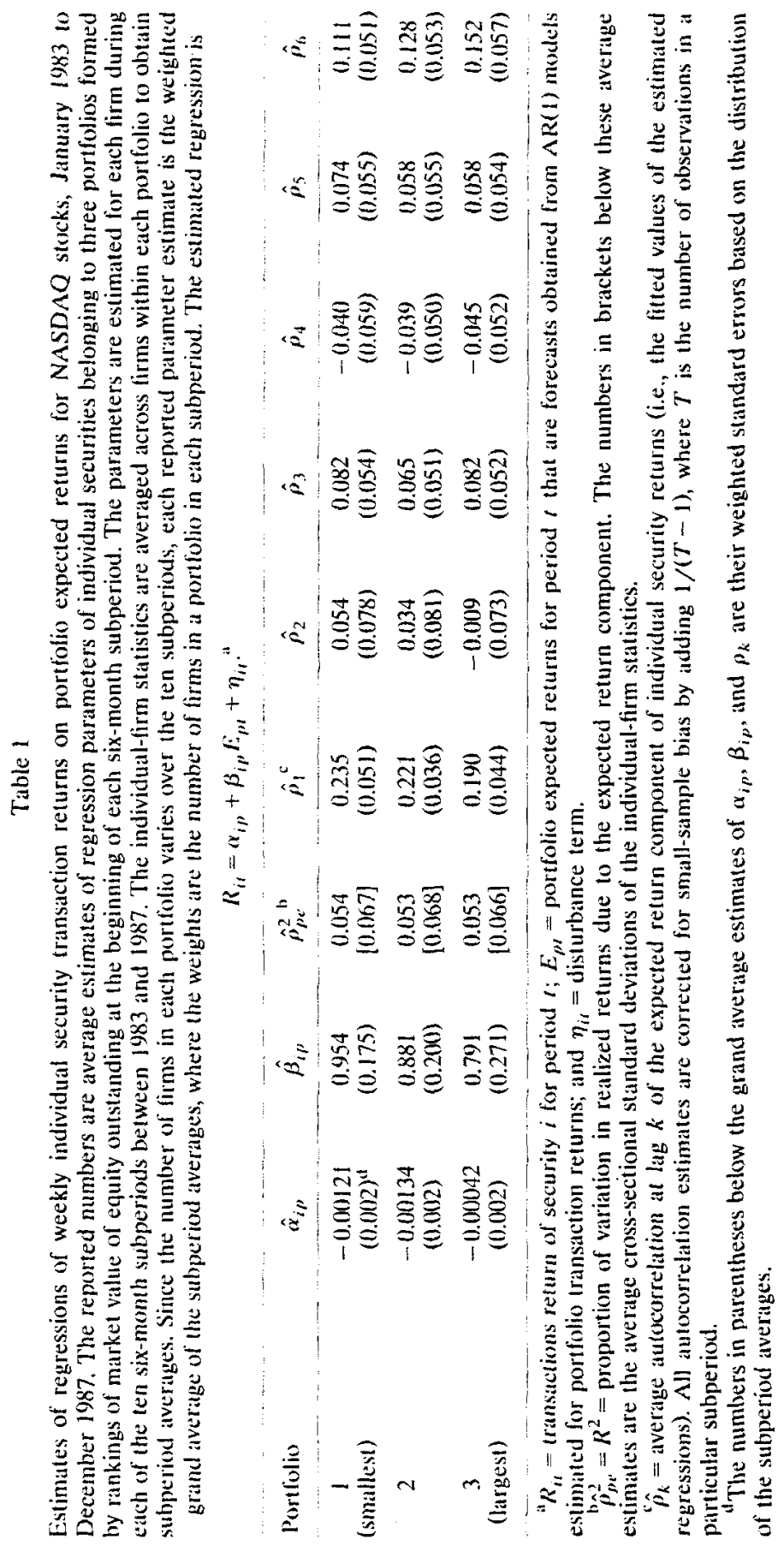


turns, $R_{p t}$. For the three NASDAQ portfolios, a stationary AR(1) model is well-specified; the residuals of the estimated models exhibit no significant autocorrelations. We use the Kalman filter approach [see Conrad and Kaul (1988)] and the Box-Jenkins technique to estimate the time-series models. The conditional forecasts obtained from both techniques use only past information, and hence estimates of $E_{p t}$ are equivalent to ex ante expected returns. We also include a dummy variable for the first week in January in the forecasting function of $E_{p t}$ to take into account the turn-of-the-year effect [see Keim (1983)]. Both the Kalman filter and the Box-Jenkins techniques provide virtually identical estimates of $E_{p t}$; we report the results based on the Box-Jenkins estimates.

The numbers in table 1 are calculated by averaging estimates across all stocks in each subperiod, and then averaging the subperiod averages. The numbers in parentheses below the grand averages are their standard errors which are based on the distribution of the subperiod averages, under the assumption that these averages are independent and identically distributed. We also report the grand averages of $\hat{\rho}_{p e}^{2}$ (that is, the $R^{2}$ 's) for each portfolio and their average cross-sectional standard deviations (in brackets). The cross-sectional standard deviations are provided simply as a measure of the cross-sectional variation in the $\hat{\rho}_{p e}^{2}$ 's.

There are several interesting aspects of the results in table 1. First, individual security returns have a significant positive relation with $E_{p t}$. The average values of the $\alpha_{i p}$ 's and $\beta_{i p}$ 's are not statistically different from zero and one, respectively, for each portfolio. However, these are not testable restrictions implied by our model, but arise (by construction) due to fixedweight portfolio returns used in the tests. Second, and perhaps more importantly, security returns also contain a positively autocorrelated expected return component that explains statistically significant proportions of their variance. The grand averages of $\hat{\rho}_{\rho e}^{2}$ exceed $5 \%$ for firms in all three portfolios. Also, the cross-sectional standard deviations of $\hat{\rho}_{p e}^{2}$ (which all exceed $6.5 \%$ ) indicate that most of the $\hat{\rho}_{p e}^{2}$ 's fall between $0 \%$ and $18 \%$.

Since the turn-of-the-year effect may reflect an anomaly rather than movements in equilibrium expected returns, we reestimate (4) using $E_{p t}$ 's which do not include the January dummy variable. The results are virtually identical to those reported in table 1: the average $R^{2}$ 's for the three portfolios are $5.2 \%, 5.2 \%$, and $5.1 \%$, compared to the reported $5.4 \%, 5.3 \%$, and $5.3 \%$. The small impact of the January effect on our results may be due to two factors: (1) small firms which experience a large January effect are not included in our sample, and (2) we have only five turn-of-the-years in our sample. Therefore, the evidence in table 1 suggests that although security returns are typically negatively autocorrelated, over $5 \%$ of their variation can be attributed to a positively autocorrelated expected return component. This analysis is similar in spirit to the one presented by Nelson and Schwert 
(1977), who show that although realized real returns on Treasury bills exhibit small autocorrelation coefficients, they can contain a highly autocorrelated expected return component.

Finally, the autocorrelations of the expected returns of firms in each portfolio are presented in the last six columns of table 1. Each autocorrelation estimate is corrected for small-sample bias by adding $1 /(T-1)$, where $T$ is the number of observations in a particular subperiod. This correction is exact under the hypothesis that expected returns are serially independent [see Moran (1948)]. The evidence shows that the expected returns of all firms, regardless of market value, are typically positively autocorrelated at all lags. Particularly noteworthy is the strong positive autocorrelation at lag 1 . Of course, these autocorrelations simply reflect the autocorrelations of portfolio expected returns, but are reported to demonstrate the likely time-series behavior of the expected returns of individual securities.

We also estimate (4) for securities on the NYSE and Amex. This sample is important because it covers a much longer period, 1962-1985, and therefore we can also estimate monthly regressions. The weekly $\hat{\rho}_{p e}^{2}$ 's range between $2.5 \%$ and $4.8 \%$, with the $1970 \mathrm{~s}$ exhibiting almost twice the degree of variation in $E_{i t}$ 's compared to the 1960 s and 1980 s. More importantly, the monthly $\hat{\rho}_{p e}^{2}$ 's are substantially higher: the average $\hat{\rho}_{p e}^{2}$ 's for small firms is $8.4 \%$ for the $1962-1985$ period and as high as $17.7 \%$ during the 1970 s.

\subsection{Asymmetry in the predictability of returns}

Our sample of NASDAQ stocks also reflects the asymmetry in the predictability of the returns of different size firms uncovered by Lo and MacKinlay (1990) and Mech (1990). Table 2, panel A, contains the first-order lagged cross-correlations for the three NASDAQ portfolios. The $i, j$ th element of the correlation matrix is the correlation between $R_{i, r-1}$ and $R_{j, t}$. Note that the elements below the diagonal are larger than those above the diagonal, with the asymmetry being more significant for the largest versus smallest firms.

An appealing property of our methodology for extracting the expected return component is that it is consistent with this intriguing asymmetry displayed by stock returns. Recall that we do not impose an equilibrium model on the expected returns of individual securities. For example, we do not assume a one-factor model [see Lo and MacKinlay (1990)] and, consequently, do not use the expected return on the market portfolio to extract $E_{i t}$ 's of individual securities, without regard to their market values. This procedure would cause expected returns of all securities to be perfectly positively correlated, and all lagged cross-correlations to be symmetric. Instead, we used expected returns of different size-based portfolios to extract 
Table 2

Weekly estimates of first-order lagged cross-correlations between (1) the transaction returns of three equally-weighted portfolios of NASDAQ stocks (panel A) and (2) the residuals of the transaction returns from models in which returns follow a stationary AR(1) process (panel B), January 1983 to December 1987. The three portfolios are formed by rankings of market value of equity outstanding at the beginning of each six-month subperiod. Under the hypothesis that the true cross-correlations are zero, the standard error of the estimated cross-correlations is about 0.062 .

\begin{tabular}{|c|c|c|c|}
\hline \multicolumn{4}{|c|}{ Panel A: Return cross-correlations ${ }^{a}$} \\
\hline Variable $(x)$ & $R_{1}$ & $R_{2 t}$ & $R_{3 t}$ \\
\hline $\begin{array}{l}R_{1, t-1} \\
R_{2, t-1} \\
R_{3 . t-1} \\
\end{array}$ & $\begin{array}{l}0.322 \\
0.324 \\
0.324\end{array}$ & $\begin{array}{l}0.235 \\
0.262 \\
0.273\end{array}$ & $\begin{array}{l}0.176 \\
0.196 \\
0.217\end{array}$ \\
\hline \multicolumn{4}{|c|}{ Panel B: Residual cross-correlations ${ }^{\mathrm{b}}$} \\
\hline Variable $(x)$ & $\hat{\varepsilon}_{1 t}$ & $\hat{\varepsilon}_{2 t}$ & $\hat{\varepsilon}_{3 t}$ \\
\hline $\begin{array}{l}\hat{\varepsilon}_{1, t-1} \\
\hat{\varepsilon}_{2, t-1} \\
\hat{\varepsilon}_{3, t-1}\end{array}$ & $\begin{array}{l}0.031 \\
0.050 \\
0.067\end{array}$ & $\begin{array}{l}0.025 \\
0.023 \\
0.043\end{array}$ & $\begin{array}{l}0.020 \\
0.014 \\
0.024\end{array}$ \\
\hline
\end{tabular}

the expected returns of securities of different market value. This procedure, in turn, takes into account the asymmetric cross-correlations.

Panel $B$ in table 2 reports first-order lagged cross-correlations between the residuals, $\hat{\varepsilon}_{t}$, from the time-series models for the portfolio returns. Note that the asymmetry is rendered insignificant. This occurs because conditional on a particular (small) portfolio's own past history of returns, the large portfolio's return contains little additional information about its future returns. In other words, by construction our portfolio expected returns exhibit the asymmetric lagged cross-correlations witnessed in realized returns. Or course, our procedure does not take into account any asymmetric lagged cross-correlations that may exist between securities within a particular size-based portfolio. However, a more complete model for expected returns of individual securities is beyond the scope of this paper.

\subsection{The bid-ask error component}

Based on our model for security prices in (1) and (2), the bid and ask prices, $B P_{i t}$ and $A P_{i t}$, of security $i$ may be written as

$$
B P_{i t}=P_{i t}-\frac{s_{i}}{2} Q_{i t}
$$


and

$$
A P_{i t}=P_{i t}+\frac{s_{i}}{2} Q_{i t}
$$

The average of the bid and ask prices will, therefore, reflect the 'true' price of security $i, P_{i t}$. Consequently, returns calculated using the average of bid and ask quotes, $R_{i r}^{\mathrm{a}}$, will reflect changes in the true value of a security (without the bid-ask bounce), so that

$$
R_{i t}^{\mathrm{a}}=E_{i t}+U_{i t} .
$$

A comparison of transaction returns and 'true' returns [see (3) and (6)] shows that the difference between them, $D R_{i t}$, will be a direct measure of the bid-ask error component,

$$
D R_{i t}=R_{i t}-R_{i t}^{\mathrm{a}}=B_{i t}=\frac{s_{i}}{2}\left[Q_{i t}-Q_{i, t-1}\right] .
$$

Most models of the bid-ask spread assume that bid-ask errors in transaction prices are independent and identically distributed [see, for example, Blume and Stambaugh (1983) and Roll (1984)]. This implies that

$$
\begin{aligned}
& \operatorname{cov}\left(B_{i t} B_{i, t-k}\right)= \begin{cases}-s_{i}^{2} / 4 & \text { if } k=1, \\
0 & \text { otherwise, }\end{cases} \\
& \operatorname{var}\left(B_{i t}\right)=s_{i}^{2} / 2,
\end{aligned}
$$

and

$$
\rho_{B k}=k \text { th-order autocorrelation of } B_{i t}= \begin{cases}-\frac{1}{2} & \text { if } k=1, \\ 0 & \text { otherwise. }\end{cases}
$$

From ( $8 \mathrm{a})-(8 \mathrm{c})$ it can be seen that $B_{i t}$ will follow an MA(1) process. Given that we have a direct measure of bid-ask errors, $D R_{i t}$, we can test whether actual bid-ask errors satisfy the above conditions.

\section{The ecidence}

Our measure of the bid-ask error component, $D R_{i t}$, will be the 'true' measure of bid-ask errors if bid/ask and transaction prices are measured synchronously. However, for the NASDAQ stocks the bid/ask (closing) quotes are measured after the (last) transaction price. This nonsynchronous measurement causes an errors-in-variables problem in $D R_{i t}$ and, 


\section{Table 3}

Average weekly estimates of autocorrelations of the bid-ask error component, $D R_{i t}$, of individual NASDAQ securities belonging to three portfolios formed by rankings of market value of equity outstanding at the beginning of each six-month subperiod. January 1983 to December 1987. Each statistic is estimated for all firms during each of the ten six-month subperiods between 1983 and 1987. The individual firm statistics are averaged across firms within each portfolio to obtain subperiod averages. Since the number of firms in each portfolio varies over the ten subperiods, each reported estimate is the weighted grand average of the subperiod averages, where the weights are the number of firms in a portfolio in each subperiod.

\begin{tabular}{ccccccc}
\hline & \multicolumn{7}{c}{ Lags } & & & \\
Portfolio & 1 & 2 & 3 & 4 & 5 & 6 \\
\hline 1 & $-0.463^{\mathrm{a}}$ & -0.002 & -0.003 & -0.007 & 0.002 & 0.002 \\
(smallest) & $(0.003)^{\mathrm{b}}$ & $(0.005)$ & $(0.004)$ & $(0.003)$ & $(0.003)$ & $(0.005)$ \\
& {$[0.152]^{\mathrm{c}}$} & {$[0.225]$} & {$[0.209]$} & {$[0.211]$} & {$[0.202]$} & {$[0.193]$} \\
2 & -0.467 & -0.003 & -0.000 & -0.005 & -0.003 & 0.005 \\
& $(0.002)$ & $(0.003)$ & $(0.003)$ & $(0.005)$ & $(0.004)$ & $(0.003)$ \\
& {$[0.153]$} & {$[0.230]$} & {$[0.218]$} & {$[0.211]$} & {$[0.200]$} & {$[0.192]$} \\
3 & -0.472 & -0.003 & 0.001 & 0.001 & -0.004 & -0.000 \\
(largest) & $(0.003)$ & $(0.005)$ & $(0.004)$ & $(0.003)$ & $(0.002)$ & $(0.002)$ \\
& {$[0.146]$} & {$[0.227]$} & {$[0.216]$} & {$[0.209]$} & {$[0.204]$} & {$[0.200]$}
\end{tabular}

${ }^{\mathrm{a}}$ The autocorrelation estimates are adjusted for small-sample bias using the following formulas [Kendall and Stuart (1976)]:

$$
\begin{aligned}
& \mathrm{E}\left(\hat{\rho}_{1}\right)=\rho_{1}+\frac{1}{T-1}\left(1+\rho_{1}\right)\left(4 \rho_{1}^{2}-2 \rho_{1}-1\right), \\
& \mathrm{E}\left(\hat{\rho}_{2}\right)=-\frac{1}{T-2}\left(1+2 \rho_{1}+2 \rho_{1}^{2}\right), \\
& \mathrm{E}\left(\hat{\rho}_{j}\right)=-\frac{1}{T-j}\left(1+2 \rho_{1}\right), \quad \forall j>2,
\end{aligned}
$$

where $\rho_{\mathrm{I}}=-0.50$ [see (8c)], and $T$ is the number of observations in a particular subperiod.

${ }^{\mathrm{b}}$ The numbers in parentheses below the grand average estimates are their weighted standard errors based on the distribution of the subperiod averages.

${ }^{c}$ The numbers in brackets are the average cross-sectional standard deviations of the individual-firm statistics.

consequently, it will overstate the importance of bid-ask errors, that is, $\operatorname{var}\left(D R_{i t}\right)>\operatorname{var}\left(B_{i t}\right)$. However, under reasonable assumptions, the autocorrelations of $D R_{i t}$ will provide unbiased estimates of the autocorrelations of the true bid-ask error component, $B_{i t}$ (see appendix).

Table 3 contains average estimates of the weekly autocorrelations (up to lag 6) of $D R_{i l}$ for firms in each portfolio. The autocorrelation estimates are adjusted for small-sample bias under the assumption that the 'true' bid-ask error component, $B_{i t}$, follows an MA(1) process with a first-order autocorrelation coefficient, $\rho_{1}$, of -0.50 [sce $(8 \mathrm{c})$ ]. The exact corrections are based on 
the following equations [Kendall and Stuart (1976)]:

$$
\begin{aligned}
& \mathrm{E}\left(\hat{\rho}_{1}\right)=\rho_{1}+\frac{1}{T-1}\left(1+\rho_{1}\right)\left(4 \rho_{1}^{2}-2 \rho_{1}-1\right), \\
& \mathrm{E}\left(\hat{\rho}_{2}\right)=-\frac{1}{T-2}\left(1+2 \rho_{1}+2 \rho_{1}^{2}\right), \\
& \mathrm{E}\left(\hat{\rho}_{j}\right)=-\frac{1}{T-j}\left(1+2 \rho_{1}\right), \quad \forall j>2 .
\end{aligned}
$$

The estimated autocorrelations in table 3 are generally consistent with the model and assumptions for $B_{i t}$. The average first-order autocorrelations of $D R_{i t}$ are similar across all portfolios, and range between -0.463 and -0.472 . All higher-order autocorrelations are small in magnitude. There is some evidence that bid-ask errors in transaction prices may not be independently distributed; the first-order autocorrelations are statistically less than 0.50 in absolute magnitude, and higher-order autocorrelations are occasionally significantly different from zero. However, the correlation in bid-ask errors in transaction prices appears to be small, and $D R_{i t}$ closely approximates an $\mathrm{MA}(1)$ process.

\section{Properties of individual security returns}

In this section, we present estimates of the degree of variation in NASDAQ weekly security returns that can be attributed to the three uncorrelated components: $E_{i t}, B_{i t}$, and $U_{i t}$. We also present evidence that reconciles the contrasting time-series behavior of short-horizon security and portfolio returns.

\subsection{Components of security returns}

Table 4 contains estimates of the proportions of variance of NASDAQ weekly security returns explained by the adjusted bid-error component, the expected return component, and the noise component. The contribution of the bid-ask error component, $B_{i t}$, to return volatility is calculated as $\operatorname{var}\left(D R_{i t}\right) / \operatorname{var}\left(R_{i t}\right)$, and is denoted by $\hat{\rho}_{p b}^{2} .{ }^{2}$ The proportion of return vari-

${ }^{2} \hat{\rho}_{\rho b}^{2}$ overstates the importance of bid-ask errors in transaction returns because bid/ask quotes are measured after transaction prices. However, since the denominator of this ratio measures the variance of weekly transaction returns, the upward bias in $\hat{\rho}_{p b}^{2}$ is likely to be small [see appendix]. Also, $\hat{\rho}_{m b}^{2}$ is calculated under the assumption that all trades occur either at the bid or the ask quote. If trades occur within the spread, $\operatorname{var}\left(D R_{i s}\right)$, and bence $\hat{\rho}_{p b}^{2}$, will overstate the importance of bid-ask errors. However, according to NASD dealers, trades within the quoted spread are infrequent and form only a small percentage of total trades in NASDAQ stocks. 


\section{Table 4}

Average weekly estimates of the proportions of variance of NASDAQ individual security transaction returns explained by the bid-ask error component, $\rho_{p b}^{2}$, the expected return component. $\rho_{p e}^{2}$, and the noise component, $\rho_{p u}^{2}$. January 1983 to December 1987 . The average estimates are of securities belonging to three portfolios formed by rankings of market value at the beginning of each six-month subperiod. Each statistic is estimated for all firms during each of the ten six-month subperiods between 1983 and 1987. The individual-firm statistics are averaged across firms within each portfolio to obtain subperiod averages. Since the number of firms in each portfolio varies over the ten subperiods, each reported estimate is the weighted grand average of the subperiod averages, where the weights are the number of firms in a portfolio in each subperiod."

\begin{tabular}{cccc}
\hline Portfolio & $\hat{\rho}_{p h}^{2}$ & $\hat{\rho}_{\rho e}^{2}$ & $\hat{\rho}_{p u}^{2}$ \\
\hline 1 & 0.190 & 0.054 & 0.756 \\
(smallest) & $(0.014)^{b}$ & $(0.004)$ & $(0.016)$ \\
& {$[0.176]^{\mathbf{c}}$} & {$[0.067]$} & {$[0.170]$} \\
2 & 0.114 & 0.053 & 0.833 \\
& $(0.007)$ & $(0.005)$ & $(0.009)$ \\
3 & {$[0.116]$} & {$[0.068]$} & {$[0.132]$} \\
(largest) & 0.058 & 0.053 & 0.889 \\
& $(0.003)$ & $(0.004)$ & $(0.005)$ \\
& {$[0.083]$} & {$[0.066]$} & {$[0.108]$}
\end{tabular}

${ }^{a} \hat{\rho}_{p b}^{2}=\operatorname{var}\left(D R_{i l}\right) / \operatorname{var}\left(R_{i l}\right)=$ proportion of variation in realized security returns due to the bid-ask error component; $\hat{\rho}_{p e}^{2}=R^{2}$ (of regression in table 1 ) = proportion of variation in realized returns due to the expected return component: and $\hat{\rho}_{p u}^{2}-1-\hat{\rho}_{p e}^{2}-\hat{\rho}_{p h}^{2}=$ proportion of variation in realized returns caused by the noise component.

${ }^{b}$ The numbers in parentheses below the grand average estimates are their weighted standard errors based on the distribution of the subperiod averages.

"The number in brackets are the averaged cross-sectional standard deviations of the individual-firm statistics.

ance explained by the expected return component, $\hat{\rho}_{p e}^{2}$, is simply the $R^{2}$ from estimates of (4). Finally, we obtain an estimate of the degree of variation in returns due to the noise component as $\hat{\rho}_{p u}^{2}=1-\hat{\rho}_{p e}^{2}-\hat{\rho}_{p b}^{2}$.

Table 4 shows that bid-ask errors induce a large degree of spurious volatility in weekly transaction returns. Average estimates of $\rho_{p b}^{2}$ range between $5.8 \%$ for large firms and $19.0 \%$ for small firms. The average cross-sectional standard deviations are also large, ranging between $8.3 \%$ and $17.6 \%$. Hence, for the small firms in our sample, between $0 \%$ and $54.2 \%$ of return volatility can typically be explained by the bid-ask error component. The proportions of return variance explained by $B_{i t}$ are also significantly larger than the proportions (of about $5 \%$ ) induced by time-varying expected returns, especially for small firms. Finally, the predominant source of variation in security returns is the arrival of new information. Estimates of the proportion of return variance explained by the rational information component range between $75.6 \%$ and $88.9 \%$. These larger proportions support the 
evidence in French and Roll (1986) and Barclay, Litzenberger, and Warner (1990).

The relative importance of the three components of most (except the very small and very large) NYSE and Amex security returns is likely to be similar to the estimates in table 4 . As noted earlier, the average quoted bid-ask spread of all firms in our sample is $2.914 \%$, which compares favorably with the average spread of $2.817 \%$ for NYSE and Amex firms at the end of 1988 . Since the spurious volatility generated by the bid-ask error component is directly related to the square of the spread [see (8b)], the importance of the bid-ask error component for the average NYSE and Amex firm is also likely to be nontrivial.

\subsection{Reconciliation of the time-series behacior of individual security and portfolio returns}

Given our model for security returns in (3) and the properties of the three components, reconciling the negative autocorrelation in security returns with the positive autocorrelation in portfolio returns is fairly straightforward. Without loss of generality, consider, for example, the first-order autocorrelation, $\rho_{i}$, of an individual security's transaction return

$$
\rho_{i}=\frac{\operatorname{cov}\left(E_{i t}, E_{i, t-1}\right)+\operatorname{cov}\left(B_{i t}, B_{i, t-1}\right)}{\operatorname{var}\left(R_{i t}\right)} .
$$

From (10) it follows that the sign of the first-order autocorrelation depends on the relative magnitudes of the autocovariances generated by the expected return and the bid-ask error components, respectively. In particular,

$$
\rho_{i} \gtreqless 0 \quad \text { iff }\left|\operatorname{cov}\left(E_{i t}, E_{i, t-1}\right)\right| \gtreqless\left|\operatorname{cov}\left(B_{i t}, B_{i, t-1}\right)\right| .
$$

The fact that the positive autocovariance generated by the expected return component is not typically discernible in the case of short-horizon security returns implies that it is contaminated by the larger negative autocovariance induced by the bid-ask error components. Of course, this need not be the case for every security. For example, the daily first-order autocorrelations of larger firms tend to be positive, though small in magnitude [Fama (1965) and French and Roll (1986)]. These findings are also consistent with our model because the autocovariance generated by bid-ask errors is likely to be smaller for larger firms, since such firms have smaller spreads [see (8a)].

Roll (1984) and Amihud and Mendelson (1987) conjecture similar explanations for their unrealistically low spread estimates based on the first-order 
autocovariance of transaction returns. Both studies assume that the expected return of a security is constant, and find that the negative first-order autocovariance is much smaller (in absolute magnitude) than that implied by the bid-ask bounce, that is, $\left|\operatorname{côv}\left(R_{i t}, R_{i t, t-1}\right)\right|<s_{i}^{2} / 4$ [see (8a)]. Roll conjectures that a positively autocorrelated expected return process could be responsible for this result. Amihud and Mendelson argue that daily return autocovariances could be small because of partial price adjustments induced by smoothing effects by market makers. However, the effects of such adjustments are likely to be dissipated over intervals as long as a week (the measurement interval used in this paper) [see Amihud and Mendelson (1987)].

We use two related tests to gauge the ability of our model to explain the differing time-series properties of individual security and portfolio returns. The first test compares the autocovariances of transaction returns with the sum of the autocovariances of our proxies for expected returns and the bid-ask error component, that is, $\operatorname{côv}\left(R_{i t}, R_{i, t-j}\right)$ versus $\operatorname{côv}\left(E_{i t}, E_{i, t-j}\right)+$ côv $\left(D R_{i t}, D R_{i, t-j}\right)$. If our model is the correct specification of the return generating process, these two estimates should be equal. However, caution must be exercised in making such comparisons since the cross-sectional averages of $\operatorname{cov}\left(E_{i t}, E_{i, t-1}\right)$ are likely to be downward biased because they measure $\operatorname{cov}\left(E_{p t}, E_{p, t-1}\right)$, which is the average of $\operatorname{cov}\left(E_{i t}, E_{j, t-1}\right), i \neq j$. Also, it is likely that the average of the true autocovariances, $\operatorname{cov}\left(E_{i t}, E_{i . t-1}\right)$, is larger than the average of $\operatorname{cov}\left(E_{i t}, E_{j, t-1}\right)$. Hence, we evaluate these estimates for general patterns, without placing undue emphasis on the exact magnitudes of $\operatorname{côv}\left(R_{i t}, R_{i, t-j}\right)$ versus côv $\left(E_{i t}, E_{i, t-j}\right)+\operatorname{côv}\left(D R_{t t}, D R_{i, t-1}\right)$.

For brevity we do not report the results, but the evidence suggests that our model is broadly consistent with most of the time-series properties of security and portfolio returns. First, our model can explain the positive first-order autocorrelation of portfolio returns versus the typically negative first-order autocorrelation in security returns. The average first-order autocovariance generated by the bid-ask error component is always larger in (absolute) magnitude than the autocovariance generated by the expected return component. Second, although our measure of $E_{i t}$ is an imperfect proxy, the patterns in its autocovariances are similar to those in the autocovariances of $R_{i t}$. For example, both $R_{i t}$ and $E_{i t}$ reflect significant autocovariances at lags 5 and 6 but, consistent with our earlier conjecture, $\operatorname{covv}\left(E_{i t}, E_{i, t-j}\right)<$ $\operatorname{côv}\left(R_{i t}, R_{i, t-j}\right)$. The only systematic characteristic of the time-series behavior of transaction returns that is inconsistent with our model are the statistically significant (but small) negative autocovariances at lag 2.

The second test is a specification test which also helps us evaluate our model's ability to explain the contrasting time-series behavior of security and portfolio transaction returns. Since we have proxies for both the expected return component, $E_{i t}$, and the bid-ask error component, $B_{i t}$, we estimate 
the following regression:

$$
R_{i t}=\alpha_{i}+\beta_{1 i} E_{i t}+\beta_{2 i} B_{i t}+\eta_{i t}
$$

The autocorrelations of the residuals of (12) provide a direct test of whether our model captures all of the components of security returns. If our model is a valid characterization of security returns, the residuals should behave like white noise, thus explaining the differing characteristics of security and portfolio returns. If bid/ask quotes and transaction prices are measured at the same time, $D R_{i t}$ would be a perfect measure of $B_{i t}$ and, by definition, $\beta_{2 i}=1$ for each security. However, due to the nonsynchronous measurement of transaction and bid/ask prices, $\operatorname{var}\left(D R_{i t}\right)>\operatorname{var}\left(B_{i t}\right)$, and $\hat{\beta}_{2 i}$ will be asymptotically downward biased. We nevertheless estimate (12) to gauge the specification of our model.

The average estimates of the parameters of (12) in table 5 show the downward bias in $\hat{\beta}_{2}$ and, as expected (see appendix), the bias is larger for larger firms. The residual autocorrelations reveal 'unexplained' patterns that are virtually identical to the autocovariance analysis of $R_{i t}, E_{i t}$, and $D R_{i t}$ discussed above. After accounting for time-varying expected returns and bid-ask errors, there remains some significant negative autocorrelation at lag 2 and significant positive autocorrelations at lags 1,5 , and 6 . The positive autocorrelations at lags 1,5 , and 6 are perfectly consistent with our model, and are a consequence of using an imperfect proxy, $E_{p t}$, to extract $E_{i t}$. On the other hand, as indicated earlier, the negative autocovariance/autocorrelation at lag 2 is not consistent with our model, especially since the bid-ask error component closely approximates an MA(1) process. However, the average magnitudes of these autocorrelations are small, ranging between -0.025 and -0.047 . Therefore, although our model does not capture all components which could potentially lead to negative autocorrelations in security returns (such as nonsynchronous trading, price discreteness, and/or market overreaction), it is unlikely that these components play an important role in the determination of 'observed' prices. Bid-ask errors appear to be the major source of negative autocorrelation in security returns.

Having established that individual security returns (though usually negatively autocorrelated) do contain a positively autocorrelated common component, our model can be readily used to explain the strong positive autocorrelation in portfolio returns. The return of a portfolio containing a large number of securities will exhibit strong positive autocovariance because bid-ask errors are cross-sectionally uncorrelated, and are diversified away in the portfolio formation process. Diversification also causes the variances of portfolio returns to be much smaller than the variances of security returns: for example, the variance of the returns of portfolio 1 is less than one-tenth 


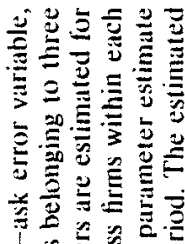

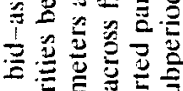

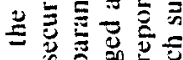

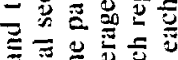

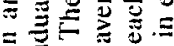

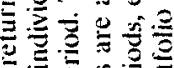

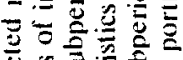

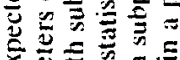

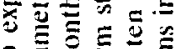

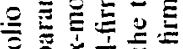

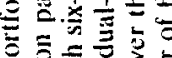

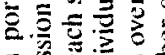

这语产.

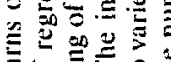

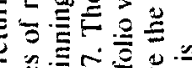

n

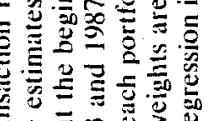

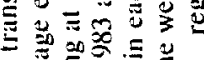

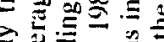

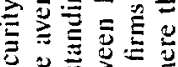

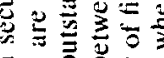

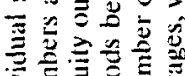

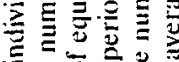

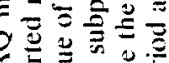

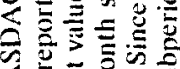

乙记造语

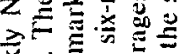

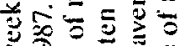

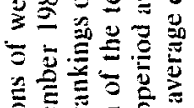

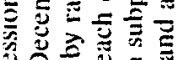

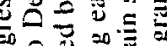

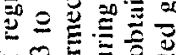

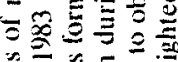

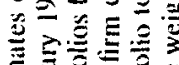

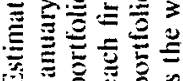

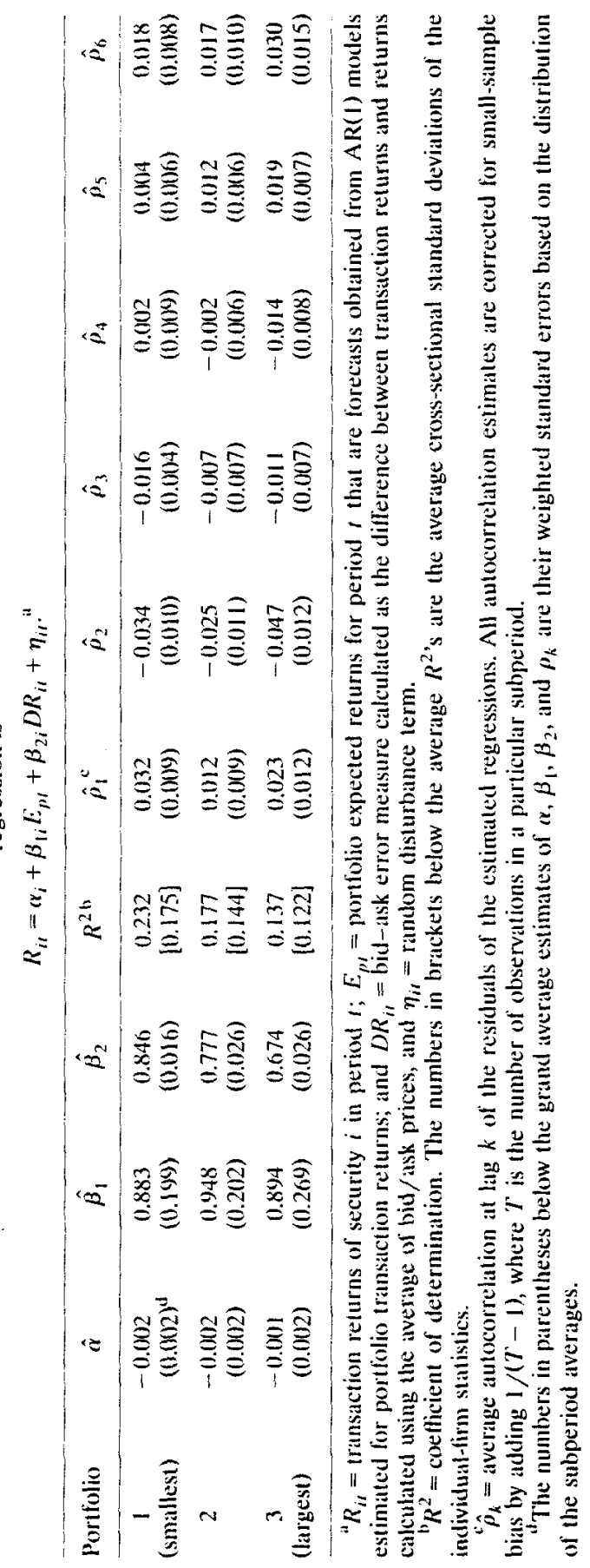


the average variance of the returns of its constituent securities. The combination of these two factors leads to strong positive autocorrelation in portfolio returns.

\section{Summary and conclusions}

In this paper, we present a simple three-component model for security returns that appears to capture the important time-series characteristics of short-horizon returns. Specifically, an individual security's return is assumed to be made up of three independent components: a positively autocorrelated expected return component, a negatively autocorrelated component induced by bid-ask errors, and a white-noise component.

We introduce a methodology to extract the 'unobservable' expected return and bid-ask error components of individual security returns, and show that these components explain substantial proportions of the variance of transaction returns - between $11 \%$ and $24 \%$ of the degree of variation in weekly transaction returns. We also reconcile the negative autocorrelation in individual security returns with the strong positive autocorrelation displayed by portfolio returns, and show that our measures of the expected return components of large versus small firms reflect the asymmetric lagged cross-correlations documented by Lo and MacKinlay (1990) and Mech (1990).

\section{Appendix: Measurement errors in the bid-ask error variable}

Recall that our bid-ask error measure, $D R_{i t}$, suffers from a nonsynchronous measurement problem because bid/ask quotes are typically measured after the transaction price. Consequently, 'measured' $D R_{i t}$ can be written as

$$
D R_{i t}=R_{i t}-R_{i t}^{\mathrm{a}}=B_{i t}+L_{i, t-1}-L_{i t} \text {, }
$$

where $L_{i t}$ is the component of 'true' return (without the bid-ask error), that is, $R_{i r}^{\mathrm{a}}$, measured over the nontrading interval between the last transaction and market close on day $t$.

Suppose $L_{i t}$ is identically distributed for a particular security [see Scholes and Williams (1977)]. From (A.1) it follows that $D R_{i t}$ will be a noisy measure of $B_{i t}$ since $\operatorname{var}\left(D R_{i t}\right)=\operatorname{var}\left(B_{i t}\right)+2 \operatorname{var}\left(L_{i t}\right)=s_{i}^{2} / 2+2 \operatorname{var}\left(L_{i t}\right)$ [see $\left.(8 \mathrm{~b})\right]^{3}{ }^{3}$ Hence, $\operatorname{var}\left(D R_{i t}\right)$ will overstate the importance of bid-ask errors in transaction returns. Although $2 \operatorname{var}\left(L_{i t}\right)$ will be small compared to the variance of

\footnotetext{
${ }^{3}$ The expression for $\operatorname{var}\left(D R_{i \ell}\right)$ is derived under the additional assumption that $L_{i,}$ is independently distributed. This is a reasonable assumption because $L_{i i}$ 's are measured over small intervals that are one week apart.
} 
'true' weekly returns, $R D_{i t}^{\mathrm{a}}$, it may be large relative to $\operatorname{var}\left(B_{i t}\right)$, which is given by $s_{i}^{2} / 2$, where $s_{i}$ is the spread of security $i$. The average values of $s_{i}^{2} / 2$ for the three NASDAQ portfolios are (approximately) $0.00136,0.00049$, and 0.00013 . Hence, especially for large firms, measurement errors in $D R_{i s}$ could have potentially important implications for specification tests of our model.

Given the assumptions about the 'true' bid-ask error component [see $(8 \mathrm{a})-(8 \mathrm{c})$ ], and the returns measured over the nontrading intervals, $L_{i t}$, it can be shown that

$$
\operatorname{cov}\left(D R_{i t}, D R_{i, t-k}\right)= \begin{cases}-s_{i}^{2} / 4-\operatorname{var}\left(L_{i t}\right) & \text { if } k=1, \\ 0 & \text { otherwise }\end{cases}
$$

and the $k$ th-order autocorrelation of $D R_{i \iota}, \rho_{k}$, is

$$
\rho_{k}= \begin{cases}-\frac{1}{2} & \text { if } k=1 \\ 0 & \text { otherwise. }\end{cases}
$$

From (A.2) and (A.3) it follows that although the first-order autocovariance of $D R_{i}$ will provide an upward-biased (in absolute magnitude) estimate of $\operatorname{cov}\left(B_{i t}, B_{i, t-1}\right)$, all its autocorrelations (including $\left.\rho_{1}\right)$ will be unbiased estimates of the autocorrelations of the 'true' bid-ask error component, $B_{i t}$.

\section{References}

Amihud, Yakov and Haim Mendelson, 1987, Trading mechanisms and stock returns: An empirical investigation, Journal of Finance 42, 533-553.

Barclay. Michael J., Robert H. Litzenberger, and Jerold B. Warner, 1990. Private information, trading volume, and stock return variances, Review of Financial Studies 3. 233-253.

Blume, Marshall E. and Robert F. Stambaugh, 1983, Biases in computed returns: An application to the size effect, Journal of Financial Economics 12, 387-404.

Conrad. Jennifer and Gautam Kaul, 1988, Time-variation in expected returns. Journal of Business $61,409-425$.

Conrad, Jennifer and Gautam Kaul, 1989, Mean reversion in short-horizon expected returns. Review of Financial Studies 2, 225-240.

Conrad. Jennifer and Gautam Kaul, 1991, Frictions and the time series properties of asset returns. Working paper (University of Michigan. Ann Arbor, MI).

Fama, Eugene F., 1965, The behavior of stock market prices, Journal of Business 38, 34-105.

Fama, Eugene $F_{,}, 1976$, Foundations of finance (Basic Books, New York, NY).

French. Kenneth R. and Richard Roll, 1986, Stock return variances: The arrival of information and the reaction of traders, Journal of Financial Economics 17, 5-26.

Gibbons, Michael R. and Wayne Ferson, 1985, Testing asset pricing models with changing expectations and an unobservable market portfolio, Journal of Financial Economics 1t, $217-236$

Kaul. Gautam and M. Nimalendran, 1990, Price reversals: Bid-ask errors or market overreaction?, Journal of Financial Economics, forthcoming.

Keim. Donald B.. 1983. Size-related anomalies and stock return seasonality: Further empirical evidence. Journal of Financial Economics 12, 13-32.

Keim, Donald B., 1989. Trading patterns, bid-ask spreads and estimated security returns: The case of common stocks at calendar turning points, Inurnal of Financial Fconomics 25, 75-97. 
Kendall, Maurice G. and Alan Stuart, 1976, The advanced theory of statistics. Vol. 3 (Griffin, London).

Lo, Andrew and A. Craig MacKinlay, 1988, Stock market prices do not follow random walks: Evidence from a simple specification test, Review of Financial Studies 1, 41-66.

Lo, Andrew and A. Craig MacKinlay, 1990, When are contrarian profits due to stock market overreaction?, Review of Financial Studies 3, 175-205.

Mech, Timothy S., 1990, The economics of lagged price adjustment. Working paper (Boston College, Boston, MA).

Moran, P.A.P., 1948, Some theorems on time series, Biometrika 35. 255-260.

Nelson, Charles R. and G. William Schwert, 1977, Short-term interest rates as predictors of inflation: On testing the hypothesis that the real rate of interest is constant, American Economic Review 67, 665-678.

Roll, Richard, 1984, A simple implicit measure of the effective bid-ask spread in an efficient market, Journal of Finance 39, 1127-1139.

Scholes, Myron and Joseph Williams, 1977, Estimating betas from nonsynchronous data, Journal of Financial Economics 5. 309-327. 